\title{
Anti-Melanogenesis Effect of Quercetin
}

\author{
Moon-Hee Choi ${ }^{1}$ and Hyun-Jae Shin ${ }^{1,2, *}$ \\ 1 Major in Cosmetic Engineering, Department of Chemical Engineering, Graduate School of Chosun University, \\ Pilmundaero, Dong-gu, Gwangju 501-759, Korea; home4134@naver.com \\ 2 Department of Biochemical and Polymer Engineering, Chosun University, Pilmundaero, Dong-gu, \\ Gwangju 501-759, Korea \\ * Correspondence: shinhj@chosun.ac.kr; Tel.: +82-62-230-7518
}

Academic Editor: Johanna Maria Gillbro

Received: 20 February 2016; Accepted: 2 May 2016; Published: 11 May 2016

\begin{abstract}
Whitening cosmetics with anti-melanogenesis activity are very popular worldwide. Many companies have tried to identify novel ingredients that show anti-melanogenesis effects for new product development. Among many plant-derived compounds, polyphenols are thought to be one of the most promising anti-melanogenesis ingredients. In order to prepare effective whitening polyphenols, 3,3,4,5,7-pentahydrosyflavone (quercetin) has been widely researched and applied to commercial products because it is present in high levels in many edible plants. Quercetin is thus a representative polyphenol and has recently gained attention in the cosmetics field. There are many controversies, however, regarding the effect of quercetin, based on in vitro studies, cell line experiments, and human trials. In this review, toxicity and efficacy data for quercetin and its derivatives in various experimental conditions (i.e., various cell lines, concentration ranges, and other parameters) were examined. Based on this analysis, quercetin itself is shown to be ineffective for hypopigmentation of human skin. However, a few types of quercetin derivatives (such as glycosides) show some activity in a concentration-dependent manner. This review provides clarity in the debate regarding the effects of quercetin.
\end{abstract}

Keywords: anti-melanogenesis; polyphenol; whitening cosmetics; hyperpigmentation; tyrosinase; quercetin

\section{Introduction}

Melanin plays a significant role in the prevention of skin damage [1]. However, the accumulation of an abnormal amount of melanin in various parts of the skin results in the development of pigmented patches that might be viewed as an aesthetic problem [2]. Excessive production of melanin and abnormal hyperpigmentation from overexposure to ultraviolet (UV) radiation may cause excessive generation of reactive species, which can lead to various skin injuries, including inflammation, age spots, melasma, and freckles [1]. In recent years, skin-whitening ingredients have become the most important components of cosmetic and hygiene products. Therefore skin-whitening ingredients that show hypo-pigmentation efficacy (i.e., anti-melanogenesis activity) are particularly important. Many researchers in academia, research institutes, and companies have attempted to identify effective and safe anti-melanogenesis and/or safe skin-whitening ingredients [3]. Skin-whitening ingredients often function via the inhibition of melanogenesis, and can also be referred to as anti-melanogenesis agents.

This review describes the fundamental synthesis of melanin, melanin signaling pathways, and the factors involved in melanogenesis and pigmentation disorders. It also examines the effects of the well-known whitening compound quercetin on the inhibition of melanogenesis. Quercetin is a representative polyphenol and has recently gained attention in the cosmetics field for its antimelanogenic properties. There are many controversies, however, regarding the effects of 
quercetin, based on in vitro studies, cell line experiments, and human trials. Specifically, it is unclear whether quercetin leads to an increase or decrease in melanin formation. Toxicity and efficacy data regarding quercetin and its derivatives for various experimental conditions (i.e., various cell lines, concentration ranges, and other parameters) are also examined in this review.

\section{Melanogenesis and Its Signal Pathway}

\subsection{Melanogenesis Mechanism and the Regulation of Melanin Biosynthesis}

Alterations to human skin, hair, and eye color are related to the type, amount, stage, and distribution of melanin [4,5]. Human skin color is determined by the outermost layer of the skin, the epidermis, where pigment-producing cells (i.e., melanocytes) are localized for melanin production. Melanin plays an important role in protecting human skin from the harmful effects of UV radiation. Upon exposure of the skin to UV radiation, melanogenesis is enhanced via the activation of the key enzyme tyrosinase, resulting in excessive production of melanin as well as DNA damage, inflammation, or other skin injuries [6-10]. Melanocytes are derived from fibroblasts in the dermis and keratinocytes in the epidermis (basal and suprabasal keratinocytes), where melanocytes transfer melanin pigments into the basal layers of the epidermis [11]. When UV radiation from sunlight exposes the skin to (photo)-oxidative stress, reactive oxygen species and reactive nitrogen species are generated, resulting in cutaneous abnormalities such as DNA-damaged epidermal hyperplasia, collagen breakdown, and inflammation. Humans naturally produce melanin pigments for photo-protection. Stem cell factor $(\mathrm{SCF})$, adrenaline noradrenaline, $\alpha$-melanocyte-stimulating hormone $(\alpha-\mathrm{MSH})$, and Wnt hormones are involved in physiological responses and interact with c-Kit, adrenergic receptors, melanocortin 1 receptor (MC1R), and Wnt receptors (Figure 1). For example, MC1R signaling regulates $3^{\prime}, 5^{\prime}$-cyclic adenosine monophosphate (cAMP) and protein kinase A (PKA), promotes cAMP-response element binding protein (CREB), and ultimately upregulates microphthalmia-associated transcription factor (MITF) in the nucleus. Upregulated MITF activates tyrosinase-related protein 1 (TRP1) in the Golgi apparatus. Subsequently, biochemical melanin synthesis (blackish-brown colored eumelanin and yellowish-red colored pheomelanin) ensues, moving these proteins to melanosomes. Melanosomes are characterized by four maturation stages and reaction directions. The combined glutathione or cysteine in DOPAquinone is converted to cysteinylDOPA or glutathionylDOPA, and pheomelanin is formed. The eumelanin stages are involved in the conversion from DOPAquinone to L-3,4-dihydroxyphenylalanine (L-DOPA) or leukodopachrome without glutathione or cysteine [12-15]. 

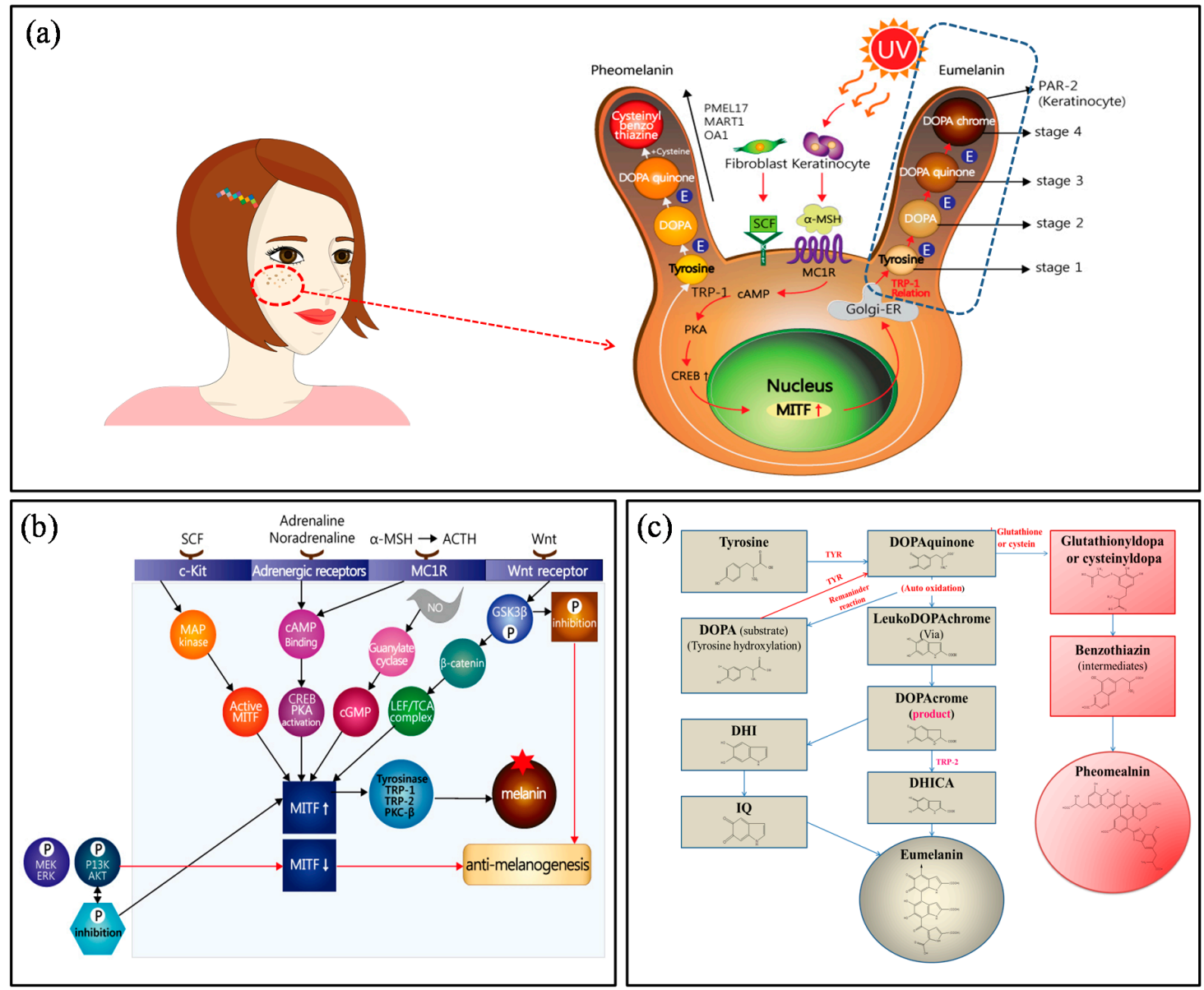

Figure 1. (a) Schematic representation of melanosome and melanin formation mechanism; (b) signal transduction pathway for anti-melanogenesis activity; and (c) chemical reaction-based melanin formation.

\subsection{Signaling Pathways Activating Melanogenesis}

To understand the signaling pathway of melanogenesis in physiological responses, four receptors must be considered. First, the c-Kit receptor is activated by SCF, followed by the activation of MAP (mitogen-activated protein) kinase and MITF. Second, adrenergic receptors bind adrenaline and noradrenaline, which activate cAMP binding, followed by CREB and PKA activation. Third, MC1R receptors are activated by $\mathrm{ACTH}$ and $\alpha-\mathrm{MSH}$, and interact with cAMP, via the identical adrenergic receptor pathway. In another pathway, nitrogen oxygen (NO) radicals activate guanylate cyclase, which activates cGMP and MITF. In particular, Wnt receptor activates GSK3 $\beta$, which promotes phosphorylation and accelerates anti-melanogenesis. The inhibition of phosphorylation in GSK3 $\beta$ increases $\beta$-catenin and the LEF/TCA complex, and activates MITF [16-19]. For the above process, activated MITF promotes the expression of tyrosinase, TRP-1 (DCT), TRP-2 (i.e., DOPA chrome tautomerase (DCT)), and PKC- $\beta$. As a result, melanin is formed. In contrast, in the extracellular signaling process, the phosphorylation of MEK/ERK and P13K/AKT downregulates MITF, leading to anti-melanogenesis effects, while the dephosphorylation process activates MITF (Figure 1b). The biochemical response for eumelanin and pheomelanin has been explained in Section 2.1.

\subsection{Inhibition of Melanogenesis through Tyrosinase Inhibition}

Every anti-melanogenesis ingredient acts via inhibitory mechanisms. Five approaches are used to inhibit melanogenesis: (1) inhibition of tyrosinase mRNA transcription; (2) aberrant tyrosinase 
maturation; (3) inhibition of tyrosinase catalytic activity; (4) acceleration of tyrosinase degradation; and (5) indirect regulation of tyrosinase activity [20]. Until now, tyrosinase has been the most common target for therapeutic agents intended to alleviate hyperpigmentation [6-9,21,22]. Multiple approaches could potentially be used to control pigmentation via the regulation of tyrosinase activity. The transcription of its mRNA, its maturation via glycosylation, its trafficking to melanosomes, as well as the modulation of its catalytic activity and/or stability are all targets for tyrosinase regulation. Well-known tyrosinase inhibitors and their sources include kojic acid, arbutin, glutathione, vitamin A (retinol), vitamin $\mathrm{B}_{3}$ (niacinamide), vitamin C, mulberry, papaya, and licorice root. Among them, the utilization of kojic acid and arbutin is still common because these agents have repeatedly been demonstrated to be effective whitening agents. The need for new kinds of natural whitening ingredients increases substantially with the growth of the market for whitening products. Some whitening products contain a mixture of many extracts known to contain tyrosinase inhibitors, but other extracts may instead act as antioxidant or anti-inflammatory agents. A number of tyrosinase inhibitors from both natural and synthetic sources have been identified. Currently, natural sources, such as polyphenols, have been gaining attention, reflecting public desire for safe and effective ingredients.

\section{Polyphenols and Melanogenesis}

\subsection{Antioxidant Activity and Melanogenesis}

The antioxidant defense system is resistant to free radicals generated in the metabolic processes [23]. Free radicals damage lipids, proteins, and DNA, and cause many diseases. Therefore, antioxidants have beneficial effects on the overall health condition of the skin and protect against UV radiation, ozone, and smoke, among other harmful substances responsible for epithelial cell and basal cell cancer and immune suppression. Representative antioxidant chemicals are plant-derived polyphenols. Polyphenols are typically divided into four primary classes: phenolic acids, flavonoids, lignans, and stilbenes (Figure 2a). Of these, phenolic acids and flavonoids are the most prevalent in nature and the most widely studied. In general, these molecules share common structural features, i.e., multiple aromatic rings and attached hydroxyl groups [24,25].

With respect to antioxidant activity, quercetin is a popular flavonoid aglycone that is found in a variety of fruits and vegetables, such as onions, curly kale, leeks, broccoli, and blueberries [24,25]. Quercetin is a potent tyrosinase inhibitor, melanogenesis inhibitor in mouse B16 melanoma cells, and an antioxidant and anticancer agent [26-30]. However, it has the opposite effect as a melanogenesis accelerator in human melanoma cells [26,31-36]. It has been shown to decrease intracellular tyrosinase activity and inhibit mushroom tyrosinase activity in a cell-free system [28,29]. In addition, quercetin inhibits melanin production in B16 melanoma cells in a dose-dependent manner [37]. However, some studies report the stimulatory effects of quercetin on cellular melanogenesis. Nagata et al. (2004) reported that quercetin enhances melanogenesis by increasing the activity and synthesis of tyrosinase in human melanoma cells and normal human melanocytes [31]. Quercetin and its derivatives also stimulate melanogenesis in the absence of $\alpha-\mathrm{MSH}$ in B16 murine melanoma cells [38]. These opposing effects of quercetin are widely debated, considering the potential application of quercetin in inhibiting tyrosinase enzyme activity in the cosmetic field. Like other flavones, quercetin contains a heterocyclic pyrone ring in its structure, which is connected on both sides to phenolic moieties. It exists in the form of rutin (quercetin-3-rutinoside), a glycoside containing a disaccharide covalently attached to the quercetin unit (Figure 2b) [34-36,38,39]. As a therapeutic agent, quercetin plays an important role in biological activities, e.g., as an anti-allergic, anti-inflammatory, anti-melanogenesis, and anti-carcinogenic agent [40-42], suggesting that it acts as a free radical scavenging agent for superoxide anions and lipid peroxyl species.

Thus, among its various biological activities, the antioxidant activity of quercetin is particularly important for anti-melanogenesis. Quercetin is considered an antioxidative agent that inactivates the tyrosinase enzyme, mediating the relationship between its antioxidant properties and anti-melanogenesis. 
It is closely associated with the free radical scavenging effect of antioxidant functions in quercetin for defense against oxidative stress. As a primary condition for anti-melanogenesis effects, the antioxidant property of polyphenols (herein, quercetin) has been extensively investigated, and these studies have focused on whether or not quercetin shows anti-melanogenesis effects.

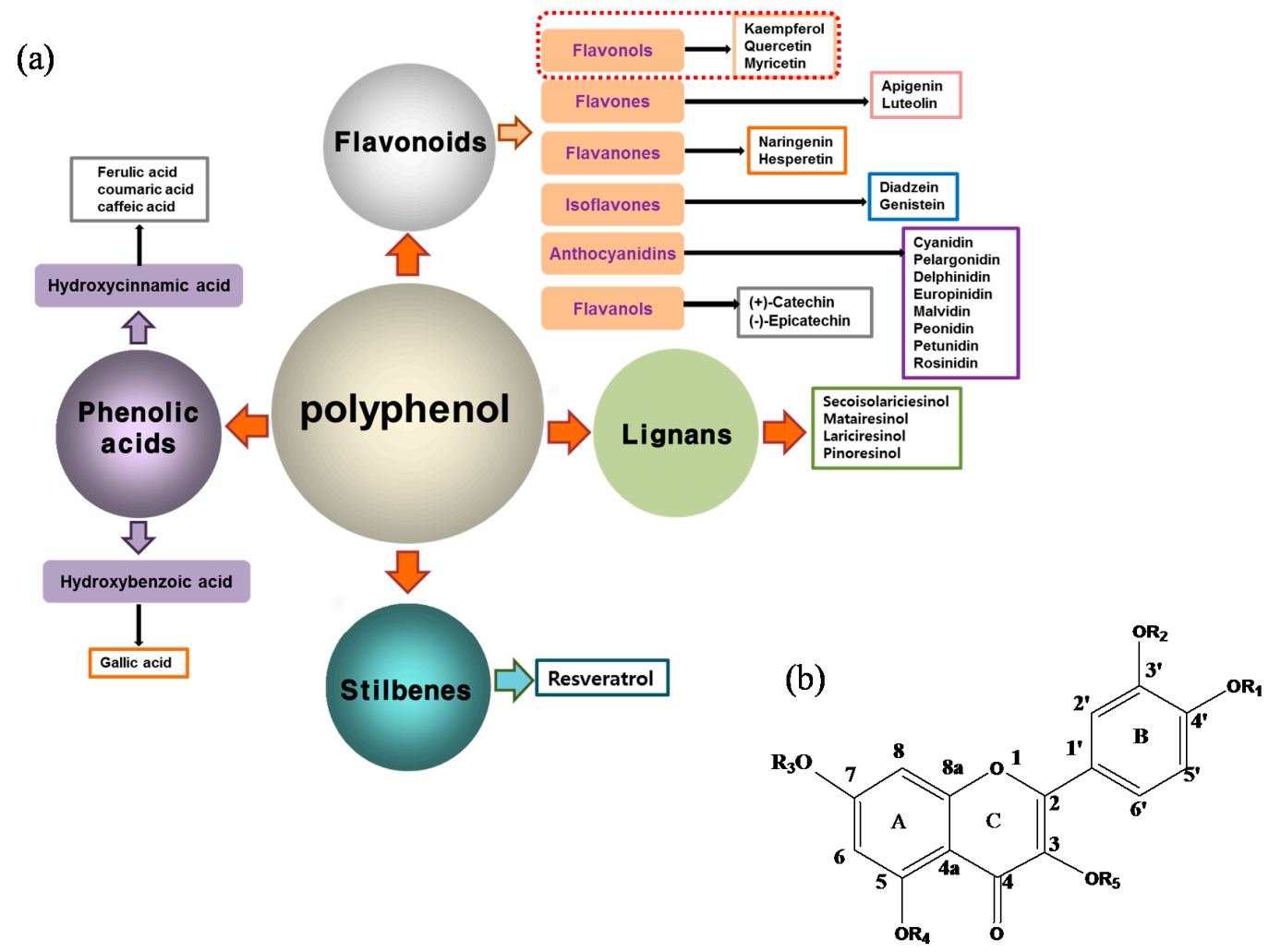

Figure 2. (a) Classification of polyphenols and (b) chemical structure of quercetin.

Anti-melanogenesis depends on the position of $\mathrm{OH}$ functional groups in the quercetin structure (Figure 2b) [34-37]. Structurally, the $\mathrm{IC}_{50}$ value for melanin inhibition by quercetin is $26.5 \mu \mathrm{M}$, while that for inhibition by quercetin- $4^{\prime}-O$-glucoside is $130.6 \mu \mathrm{M}$. However, the cell viabilities for quercetin and quercetin- $4^{\prime}$-O-glucoside are $88 \%$ and $82 \%$, respectively. Quercetin-3, $4^{\prime}$ - $O$-diglucoside and quercetin-3,4'-O-rhamnoside (rutin) are safe because they have high antioxidant activity with a related B ring, but have little effect on hypo-pigmentation. Thus, although quercetin and quercetin- $4^{\prime}$-O-glucoside have the ability to reduce melanin content, they also demonstrate cell toxicity [43]. Conclusively, $C 4^{\prime}$ glycosylation of the quercetin structure doubles its antioxidant activity but decreases the inhibition of melanogenesis five-fold. Quercetin presents a dilemma with respect to skin-whitening agents; its safety should be considered for practical human applications. In addition, $4^{\prime}$-O- $\beta$-D-glucopyranosyl-quercetin-3-O- $\beta$-D-glucopyranosyl- $(1 \rightarrow 4)$ - $\beta$-D-glucopyranoside shows an increase in melanin content by MITF expression with no cytotoxic effects at $10 \mu \mathrm{M}$, suggesting that the C4' site plays a critical role in melanogenesis [35]. Yamauchi et al. (2013) determined that R5 substituted with $-\mathrm{OCH}_{3}$ groups stimulates melanogenesis activity, and both $\mathrm{R} 3$ and $\mathrm{R} 5$ substituted with $-\mathrm{OCH}_{3}$ groups result in a cell viability of $\sim 60 \%$ at $12.5 \mu \mathrm{M}$ [35]. These observations support the role of $\mathrm{C} 4$ in melanogenesis. In an expanded analysis, Yamauchi et al. (2014) reported that synthesized quercetin glycosides have an anti-melanogenesis effect and demonstrate less cell toxicity for $\mathrm{R}_{1}=$ cellobiose, $\mathrm{R}_{3}=\mathrm{OH}, \mathrm{R}_{5}=$ cellobiose, or $\mathrm{R}_{1}=\mathrm{OH}, \mathrm{R}_{3}=$ cellobiose, $\mathrm{R}_{5}=$ cellobiose, or $\mathrm{R}_{1}=$ glucose, $\mathrm{R}_{3}=\mathrm{OH}$, $\mathrm{R}_{5}=$ glucose [34]. Other quercetin glycosides show either no anti-melanogenesis effect (or the acceleration of melanogenesis), cell toxicity, or their combination. Therefore, quercetin glycosides are not suitable for use as whitening agents [36]. 


\subsection{In Vitro and in Vivo Anti-Melanogenesis Effects of Quercetin}

Most anti-melanogenesis experiments using quercetin have been in vitro, rather than in vivo, assays. The results for cellular tests of melanin content, tyrosinase activity, mRNA expression, and protein level expression based on in vitro investigations of quercetin and its derivatives, including compounds derived from natural extracts, are summarized in Table 1. Generally, B16F10/B16F1 melanoma cells and mouse melanoma cells have been utilized, but human melanoma of vagina (HMVII) cells and normal human epidermal melanocytes (NHEM) have also been used, though infrequently. Depending on the concentration of quercetin or its compounds in natural extracts, at low concentrations, in the range of 10-20 $\mu \mathrm{M}$, melanin content in cells increases, while at concentrations of $20-50 \mu \mathrm{M}$, it decreases. At a concentration in the range of 50-100 $\mu \mathrm{M}$, melanin content decreases and cytotoxicity increases (Figure 3). By contrast, at concentrations of 10-500 $\mu \mathrm{M}$, the melanin content increases; however, these concentrations are safe with respect to cell viability. This means that natural quercetin compounds show less cell toxicity. However, importantly, those compounds are not effective in reducing melanin content. Furthermore, as shown in Table 1, Nagata et al. found that tyrosinase activity in cell systems is enhanced, but there is no effect on mRNA expression, resulting in the overexpression of the tyrosinase protein owing to quercetin treatment at concentrations of 1-20 $\mu \mathrm{M}$ [31]. In a cell-free system, tyrosinase activity is inhibited at 10-100 $\mu \mathrm{M}$. Interestingly, in a cell system, tyrosinase activity increased at 5-10 $\mu \mathrm{M}$, but was inhibited at 20-50 $\mu \mathrm{M}$. Tyrosinase, TRP-1, and TRP-2 show different protein expression patterns for the tested quercetin concentrations. Specifically, at quercetin concentrations of 10 and $20 \mu \mathrm{M}$, tyrosinase is overexpressed, but at $50 \mu \mathrm{M}$, it is expressed at slightly lower levels. At quercetin concentrations of 10, 20, and $50 \mu \mathrm{M}$, TRP-1 shows gradually lower expression, while at 5 and $10 \mu \mathrm{M}$, TRP-2 is expressed, and at 20 and $50 \mu \mathrm{M}$, its expression gradually decreases [41]. In particular, Takekoshi et al. (2013) reported an increase in melanin content at quercetin concentrations $>50 \mu \mathrm{M}$ [26]. Tyrosinase and TRP-2 are overexpressed at quercetin concentrations of $5-160 \mu \mathrm{M}$, but there is no effect on TRP-1 at quercetin concentrations of 50-160 $\mu \mathrm{M}$. Likewise, the same group showed that at $10 \mu \mathrm{M}$ quercetin, melanin content increases and tyrosinase is overexpressed after three days [21].

For quercetin in the Capparis spinosa L. extract, the melanin content increases at 50-500 $\mu \mathrm{M}$ and tyrosinase is expressed at $300 \mu \mathrm{M}$ after $24 \mathrm{~h}$ [33]. Masuda et al. (2012) showed that at 12.5-50 $\mu \mathrm{M}$, the melanin content increases, while at 100 and $200 \mu \mathrm{M}$, the melanin content decreases [44]. In a cell system, tyrosinase activity was enhanced at quercetin concentrations of $200 \mu \mathrm{M}$. As P-38 MAPK is overexpressed, MITF is reduced, and ERK1/2 is overexpressed, but the activation of MITF results in low expression of tyrosinase at the protein level quercetin concentrations of 5 and $200 \mu \mathrm{M}$ [44]. Quercetin extracted from rosehip (Rosa canina L.) results in a decrease in melanin content at $20 \mu \mathrm{M}$ and tyrosinase activity in both cell and cell-free systems is inhibited at $10-40 \mu \mathrm{M}$, resulting in low tyrosinase expression at the protein level at these concentrations [37]. An et al. (2008) tested quercetin derivatives with taxifolin and luteolin as additives, and the melanin content decreased at $200 \mu \mathrm{M}$ [39]. In the presence of taxifolin and luteolin in a cell system, tyrosinase activity is inhibited and tyrosinase is overexpressed at quercetin concentration of $200 \mu \mathrm{M}$ [38]. Synthesized quercetin is related to decreased melanin content at concentrations of $6.25-100 \mu \mathrm{M}$. It is associated with low expression of tyrosinase, TRP-1, TRP-2, and p38 MAPK and a lack of stimulation of MITF and phosphorylated-p38 (p-p38) MAPK [34]. Most quercetin derivatives lead to increased melanin content. However, quercetin-galactose-rhamnose-xylose and quercetin-glucose-rhamnose result in decreases at $72 \mu \mathrm{M}$ after $72 \mathrm{~h}$ and the mRNA expression levels of tyrosinase, TRP-1, TRP, MITF, and MC1R are downregulated. As a result, the tyrosinase protein expression level is low. Arung et al. (2011) found that quercetin glucoside (Allium cepa) decreases the melanin content at 1-100 $\mu \mathrm{M}$ [43]. 
Table 1. Literature summary of in vitro anti-melanogenesis effect by quercetin and its derivatives.

\begin{tabular}{|c|c|c|c|c|c|c|c|c|}
\hline Quercetin Type (Pure or Derivatives) & Cell Line & $\begin{array}{l}\text { Concentration } \\
\text { Range }\end{array}$ & Cell Viability Conc. & Melanin Content & $\begin{array}{c}{ }^{*} \text { Tyrosinase } \\
\text { Activity (Cell Free } \\
\text { or Cell System) }\end{array}$ & $\begin{array}{c}\text { mRNA } \\
\text { Expression }\end{array}$ & $\begin{array}{l}\text { Protein Level } \\
\text { Expression }\end{array}$ & Reference \\
\hline Quercetin $\left(3,3^{\prime}, 4^{\prime}, 5,7\right.$-pentahydroxyflavone) & $\begin{array}{l}\text { 1. Human } \\
\text { melanoma of } \\
\text { vagina II (HMVII) } \\
\text { melanoma cell } \\
\text { 2. Normal human } \\
\text { epidermal } \\
\text { melanocytes } \\
\text { (NHEM) }\end{array}$ & $\begin{array}{c}\text { 1, 5, 10, and } \\
20 \mu \mathrm{M}\end{array}$ & $\begin{array}{l}1 . \leqslant 10 \mu \mathrm{M} \text { for } \\
\text { HMVII-No } \\
\text { cytotoxicity } \\
2 . \leqslant 1 \mu \mathrm{M} \text { for } \\
\text { NHEM-No } \\
\text { cytotoxicity }\end{array}$ & $\begin{array}{l}\leqslant 20 \mu \mathrm{M} \text {-Increase } \\
\text { Blocking of tyrosinase } \\
\text { activation by } \\
\text { quercetin } \\
\text { 1. Cycloheximide } \\
(10 \mu \mathrm{M}) \\
\text { 2. actinomycin-D } \\
(5 \mu \mathrm{M})\end{array}$ & $\begin{array}{c}\text { Tyrosinase activity } \\
\text { (cell system) } \\
\text { At } 1-20 \mu \mathrm{M} \\
\text {-Enhance }\end{array}$ & $\begin{array}{c}\text { Tyrosinase-No } \\
\text { effect }\end{array}$ & $\begin{array}{c}\text { Tyrosinase At } \\
1-20 \\
\mu \mathrm{M}-\text { Overexpression }\end{array}$ & $\begin{array}{l}\text { Nagata et al., } \\
\text { (2004) [31] }\end{array}$ \\
\hline Quercetin & $\begin{array}{l}\text { B16F10 melanoma } \\
\text { (mice) NHEM } \\
\text { (human) }\end{array}$ & $10-100 \mu \mathrm{M}$ & $>20 \mu \mathrm{M}-$ Cell toxicity & $\begin{array}{c}5 \text { and } 10 \mu \mathrm{M} \\
72 \mathrm{~h}-\text { Increase }\end{array}$ & $\begin{array}{c}\text { Tyrosinase activity } \\
\text { (cell free system) } \\
\text { At 10-100 } \\
\mu \mathrm{M} \text {-Inhibit } \\
\text { Tyrosinase Activity } \\
\text { (cell system) } \\
\text { At 5-10 } \\
\mu \mathrm{M}-\text { Enhance } \\
\text { At 20-50 } \\
\mu \mathrm{M} \text {-Inhibit }\end{array}$ & $\begin{array}{l}\text { p-PKA At 10-50 } \\
\mu \mathrm{M}-\text { Gradually } \\
\text { low expression }\end{array}$ & $\begin{array}{c}\text { Tyrosinase At } 10 \text { and } \\
20 \mu \mathrm{M} \\
\text {-Overexpression } \\
\text { At } 50 \mu \mathrm{M}-\text { Slightly low } \\
\text { expression } \\
\text { TRP-1 At } 10,20 \text {, and } \\
50 \mu \mathrm{M} \text {-Gradually low } \\
\text { expression } \\
\text { TRP-2 At } 5 \text { and } \\
10 \mu \mathrm{M} \\
\text {-Overexpression At } 20 \\
\text { and } 50 \mu \mathrm{M}-\text { Gradually } \\
\text { low expression }\end{array}$ & $\begin{array}{c}\text { Yang et al., (2011) } \\
\text { [41] }\end{array}$ \\
\hline Quercetin & $\begin{array}{l}\mathrm{C} 3 \mathrm{H} / \mathrm{HeN} \text { Jrc mice } \\
\text { (hair follicle tissues) }\end{array}$ & $50-160 \mu \mathrm{M}$ & - & $>50 \mu \mathrm{M}$-Increase & - & - & $\begin{array}{c}\text { Tyrosinase TRP-2 At } \\
5-160 \mu \mathrm{M} \\
\text {-Overexpression } \\
\text { TRP-1 At } \\
50-160 \mu \mathrm{M}-\text { No effect }\end{array}$ & $\begin{array}{l}\text { Takekoshi et al., } \\
\text { (2013) [26] }\end{array}$ \\
\hline Quercetin & HMVII (human) & $1-20 \mu \mathrm{M}$ & $\begin{array}{l}\leqslant 20 \mu \mathrm{M}-\mathrm{No} \\
\text { cytotoxicity }\end{array}$ & $\leqslant 20 \mu \mathrm{M}$-Increase & $\begin{array}{c}\text { Tyrosinase Activity } \\
\text { (cell system) At } 20 \\
\mu \mathrm{M}-\text { Enhance }\end{array}$ & - & - & $\begin{array}{l}\text { Takekoshi et al., } \\
\text { (2014) [21] }\end{array}$ \\
\hline Quercetin & $\begin{array}{l}\text { Melanoderm } \\
\text { (human) }\end{array}$ & $10-100 \mu \mathrm{M}$ & $\begin{array}{l}\leqslant 100 \mu \mathrm{M}-\mathrm{No} \\
\text { cytotoxicity }\end{array}$ & At $10 \mu \mathrm{M}$-Increase & $\begin{array}{c}\text { Tyrosinase Activity } \\
\text { (cell system) } \\
\text { At } 10 \mu \mathrm{M} 7 \\
\text { day-Enhance } \\
\end{array}$ & - & $\begin{array}{c}\text { Tyrosinase After } 3 \text { days } \\
\text { at } 10 \mu \mathrm{M} \\
\text { —Overexpression }\end{array}$ & $\begin{array}{l}\text { Takeyama et al., } \\
\text { (2004) [32] }\end{array}$ \\
\hline Quercetin & $\begin{array}{l}\text { B16F10 melanoma } \\
\text { Cell (mice) }\end{array}$ & $\begin{array}{c}\text { 5, 10, and } \\
50 \mu \mathrm{M}\end{array}$ & $>10 \mu \mathrm{M}-$ Cell toxicity & $\begin{array}{c}\text { At } 5,10 \text {, and } \\
50 \mu \mathrm{M} \text {-Decrease }\end{array}$ & - & - & - & $\begin{array}{c}\text { Kim et al., (2012) } \\
\text { [27] }\end{array}$ \\
\hline Quercetin & Melan-a cells (mice) & $\begin{array}{l}0.01-60 \\
\mu \mathrm{g} / \mathrm{mL}\end{array}$ & $\begin{array}{c}0.01-60 \mu \mathrm{g} / \mathrm{mL}-\mathrm{No} \\
\text { cytotoxicity }\end{array}$ & $60 \mu \mathrm{g} / \mathrm{mL}$-Decrease & - & - & - & $\begin{array}{c}\text { Chun et al., (2002) } \\
{[28]}\end{array}$ \\
\hline
\end{tabular}


Table 1. Cont

\begin{tabular}{|c|c|c|c|c|c|c|c|c|}
\hline Quercetin Type (Pure or Derivatives) & Cell Line & $\begin{array}{l}\text { Concentration } \\
\text { Range }\end{array}$ & Cell Viability Conc. & Melanin Content & $\begin{array}{c}\text { * Tyrosinase } \\
\text { Activity (Cell Free } \\
\text { or Cell System) }\end{array}$ & $\begin{array}{c}\text { mRNA } \\
\text { Expression }\end{array}$ & $\begin{array}{l}\text { Protein Level } \\
\text { Expression }\end{array}$ & Reference \\
\hline Quercetin & $\begin{array}{l}\text { B16F10 melanoma } \\
\text { (mice) }\end{array}$ & $30-50 \mu \mathrm{g} / \mathrm{mL}$ & $\begin{array}{c}>30 \mu \mathrm{g} / \mathrm{mL} \text { for } 48 \mathrm{~h} \\
\text { culture with MTT } \\
\text { assay-Cell toxicity } \\
>2.5 \mu \mathrm{g} / \mathrm{mL} \text { for } 48 \mathrm{~h} \\
\text { culture with Trypan } \\
\text { blue test-Cell } \\
\text { toxicity }\end{array}$ & $\begin{array}{c}30-100 \\
\mu \mathrm{g} / \mathrm{mL}-\text { Decrease }\end{array}$ & - & - & - & $\begin{array}{c}\text { Chun et al., (2002) } \\
\text { [29] }\end{array}$ \\
\hline Quercetin & - & $0-200 \mu \mathrm{M}$ & - & $<200 \mu \mathrm{M}$-Decrease & - & - & - & $\begin{array}{l}\text { Xie et al., (2003) } \\
\text { [22] }\end{array}$ \\
\hline Quercetin (Capparis spinosa L. extract) & $\begin{array}{l}\text { B16 melanoma cells } \\
\text { (mice) }\end{array}$ & $5-500 \mu \mathrm{M}$ & $\begin{array}{l}\text { Up to } 500 \mu \mathrm{M}-\mathrm{No} \\
\text { cytotoxicity }\end{array}$ & $\begin{array}{c}\text { At } 50-500 \\
\mu \mathrm{M} \text {-Increase }\end{array}$ & - & $\begin{array}{c}\text { Tyrosinase, } \\
\text { TRP-1 } \\
\text { TRP-2—not } \\
\text { determined } \\
\end{array}$ & $\begin{array}{l}\text { Tyrosinase After } 24 \mathrm{~h} \text { at } \\
300 \mu \mathrm{M} \text {-Increase }\end{array}$ & $\begin{array}{l}\text { Matsuyama et al., } \\
\text { (2009) [33] }\end{array}$ \\
\hline Quercetin (Morinda citrifolia extract) & $\begin{array}{l}\text { B16 melanoma cells } \\
\text { (mice) }\end{array}$ & $\begin{array}{l}12.5,25,50 \\
100, \text { and } \\
200 \mu \mathrm{M}\end{array}$ & - & $\begin{array}{c}12.5-50 \mu \mathrm{M}-\text { Increase } \\
100 \text { and } \\
200 \mu \mathrm{M} \text {-Decrease }\end{array}$ & $\begin{array}{c}\text { Tyrosinase activity } \\
\text { (cell system) At } 200 \\
\mu \mathrm{M}-\text { Enhance }\end{array}$ & $\begin{array}{l}\text { p-p38 MAPK- } \\
\text { Overexpression } \\
\text {-Depression of } \\
\text { MITF ERK1/2- } \\
\text { Overexpression } \\
\text {-Activation of } \\
\text { MITF }\end{array}$ & $\begin{array}{l}\text { Tyrosinase At } 5 \text { and } 200 \\
\mu \mathrm{M} \text {-Low expression }\end{array}$ & $\begin{array}{l}\text { Masuda et al., } \\
\text { (2012) [44] }\end{array}$ \\
\hline Quercetin rose hip (Rosa canina L. extract) & $\begin{array}{l}\text { B16 melanoma cells } \\
\text { (mice) }\end{array}$ & $\begin{array}{c}\text { Eluates } 250 \\
\mu \mathrm{g} / \mathrm{mL} \text { and } \\
20 \mu \mathrm{M} \text { from } \\
\text { EtOAC } \\
\text { fraction }\end{array}$ & $\begin{array}{l}\text { At } 250 \mu \mathrm{g} / \mathrm{mL}-\mathrm{No} \\
\text { cytotoxicity At } 20 \\
\mu \mathrm{M}-\text { No cytotoxicity }\end{array}$ & $\begin{array}{c}\text { At } 250 \mu \mathrm{g} / \mathrm{mL} \\
\text {-Decrease At } 20 \\
\mu \mathrm{M} \text {-Decrease }\end{array}$ & $\begin{array}{c}\text { * Tyrosinase activity } \\
\text { (cell free system \& } \\
\text { cell system) at } \\
10-40 \mu \mathrm{M} \\
\text { - Inhibit }\end{array}$ & - & $\begin{array}{l}\text { Tyrosinase } 10-40 \mu \mathrm{M} \\
\text { - Low expression }\end{array}$ & $\begin{array}{c}\text { Fujii et al., (2009) } \\
\text { [37] }\end{array}$ \\
\hline Quercetin (Anastatica hierochuntica) & $\begin{array}{l}\text { B16 melanoma } 4 \text { A5 } \\
\text { cells (mice) }\end{array}$ & $0-100 \mu \mathrm{M}$ & $\begin{array}{l}\text { At }<100 \mu \mathrm{M}-\text { No } \\
\quad \text { cytotoxicity }\end{array}$ & At $<30 \mu \mathrm{M}$-Decrease & $\begin{array}{c}\text { Tyrosinase activity } \\
\text { (cell free system) at } \\
1-30 \mu \mathrm{M} \\
\text {-Enhance }\end{array}$ & $\begin{array}{l}\text { Tyrosinase } \\
\text { TRP-1 TRP-2 } \\
\text { - Low } \\
\text { expression }\end{array}$ & - & $\begin{array}{l}\text { Nakashima et al., } \\
\text { (2010) [45] }\end{array}$ \\
\hline & $\begin{array}{l}\text { B16F10 melanoma } \\
\text { cells (mice) }\end{array}$ & $0-200 \mu \mathrm{M}$ & $\begin{array}{l}0-200 \mu \mathrm{M}-\text { Cell } \\
\text { toxicity }\end{array}$ & $\begin{array}{l}\text { At } 200 \mu \mathrm{M} \\
\text {-Decrease }\end{array}$ & $\begin{array}{l}\text { Tyrosinase Activity } \\
\text { by Taxifolin and } \\
\text { Luteolin (cell } \\
\text { system) At } 200 \mu \mathrm{M} \\
\text {-Inhibit }\end{array}$ & - & $\begin{array}{l}\text { Tyrosinase by Taxifolin } \\
\text { and Luteolin At } 200 \\
\mu \mathrm{M}-\text { Overexpression }\end{array}$ & $\begin{array}{l}\text { An et al., (2008) } \\
\text { [39] }\end{array}$ \\
\hline
\end{tabular}


Table 1. Cont.

\begin{tabular}{|c|c|c|c|c|c|c|c|}
\hline Quercetin Type (Pure or Derivatives) & $\begin{array}{l}\text { Concentration } \\
\text { Range }\end{array}$ & Cell Viability Conc. & Melanin Content & $\begin{array}{c}{ }^{*} \text { Tyrosinase } \\
\text { Activity (Cell Free } \\
\text { or Cell System) }\end{array}$ & $\begin{array}{c}\text { mRNA } \\
\text { Expression }\end{array}$ & $\begin{array}{l}\text { Protein Level } \\
\text { Expression }\end{array}$ & Reference \\
\hline $\begin{array}{l}\text { 2. 3', } \text { 3, }^{\prime},-\mathrm{O} \text {-trimethylquercetin (Synthesized } \\
\text { quercetin) }\end{array}$ & - & $\begin{array}{c}\text { From at } 6.25 \\
\mu \mathrm{M}-\text { Cell toxicity }\end{array}$ & $\begin{array}{l}\text { At } 6.25-100 \mu \mathrm{M} \\
\text {-Increase }\end{array}$ & - & - & $\begin{array}{l}\text { Tyrosinase TRP-1 TRP-2 } \\
\text { p38 MAPK-Low } \\
\text { expression At 3.1-12.5 } \\
\text { } \text { M MITF p-p38 } \\
\text { MAPK-Lack of } \\
\text { stimulation }\end{array}$ & $\begin{array}{l}\text { Yamauchi et al., } \\
\text { (2014) [36] }\end{array}$ \\
\hline 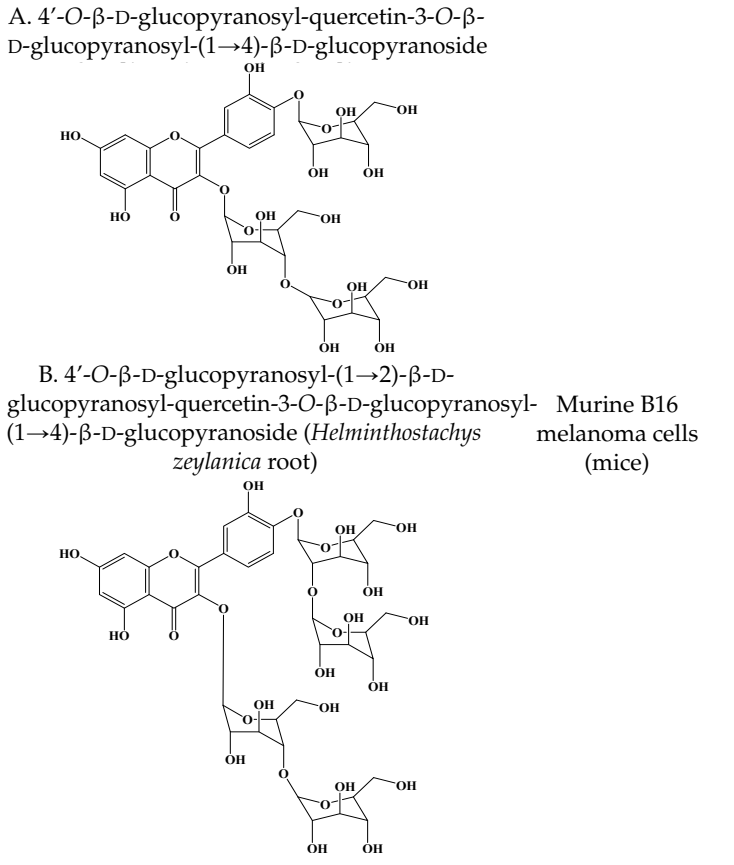 & - & $\begin{array}{l}\text { At } 10 \mu \mathrm{M}-\mathrm{No} \\
\text { cytotoxicity }\end{array}$ & At $10 \mu \mathrm{M}$-Increase & 更 & - & 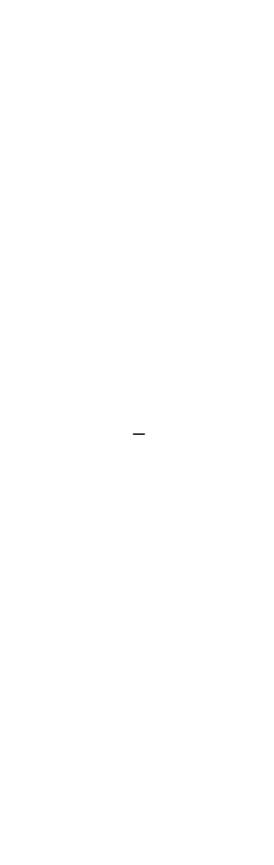 & 更 \\
\hline
\end{tabular}


Table 1. Cont.

\begin{tabular}{|c|c|c|c|c|c|c|c|c|}
\hline Quercetin Type (Pure or Derivatives) & Cell Line & $\begin{array}{l}\text { Concentration } \\
\text { Range }\end{array}$ & Cell Viability Conc. & Melanin Content & $\begin{array}{c}{ }^{*} \text { Tyrosinase } \\
\text { Activity (Cell Free } \\
\text { or Cell System) }\end{array}$ & $\begin{array}{c}\text { mRNA } \\
\text { Expression }\end{array}$ & $\begin{array}{l}\text { Protein Level } \\
\text { Expression }\end{array}$ & Reference \\
\hline $\begin{array}{l}\text { Quercetin glucosides }(4.5 \%) \\
\text { (Wine flavonoid fraction) }\end{array}$ & Melan-a cells (mice) & - & $\begin{array}{l}\text { At } 200 \mathrm{mg} / \mathrm{L}-\mathrm{Cell} \\
\text { toxicity }\end{array}$ & At $8 \mathrm{mg} / \mathrm{L}$-Increase & $\begin{array}{l}\text { Tyrosinase activity } \\
\text { (cell system) at } 40 \\
\text { and } 200 \mu \mathrm{M} \\
\text { - Inhibit }\end{array}$ & - & - & $\begin{array}{l}\text { Gómez-Cordovés } \\
\text { et al., (2001) [40] }\end{array}$ \\
\hline B. Quercetin-3, & $\begin{array}{l}\text { B16 melanoma cell } \\
\text { (mice) }\end{array}$ & - & $\begin{array}{c}>10 \mu \mathrm{M}-\text { Slight cell } \\
\text { toxicity }\end{array}$ & $\begin{array}{l}\text { At } 10,100, \text { and } 200 \\
\mu \mathrm{M} \text {-Increase }\end{array}$ & $\begin{array}{c}\text { Tyrosinase activity } \\
\text { (cell system) At 50, } \\
100 \text {, and } 200 \\
\mu \mathrm{M}-\text { Slight increase }\end{array}$ & - & - & $\begin{array}{c}\text { Yamauchi et al., } \\
\text { (2014) [34] }\end{array}$ \\
\hline $\begin{array}{l}\text { Quercetin 3-O- } \beta \text {-D-galactopyranoside } \\
\text { (Nelnumbo nucifera Gaertn.) } \\
\text { он }\end{array}$ & - & - & - & - & $\begin{array}{l}\text { Tyrosinase activity } \\
\text { (cell free system) } \\
\mathrm{IC}_{50}=15.67 \mu \mathrm{g} / \mathrm{mL}\end{array}$ & - & - & $\begin{array}{c}\text { Cho et al., (2013) } \\
\text { [30] }\end{array}$ \\
\hline
\end{tabular}


Cosmetics 2016, 3,18

11 of 16

Table 1. Cont.

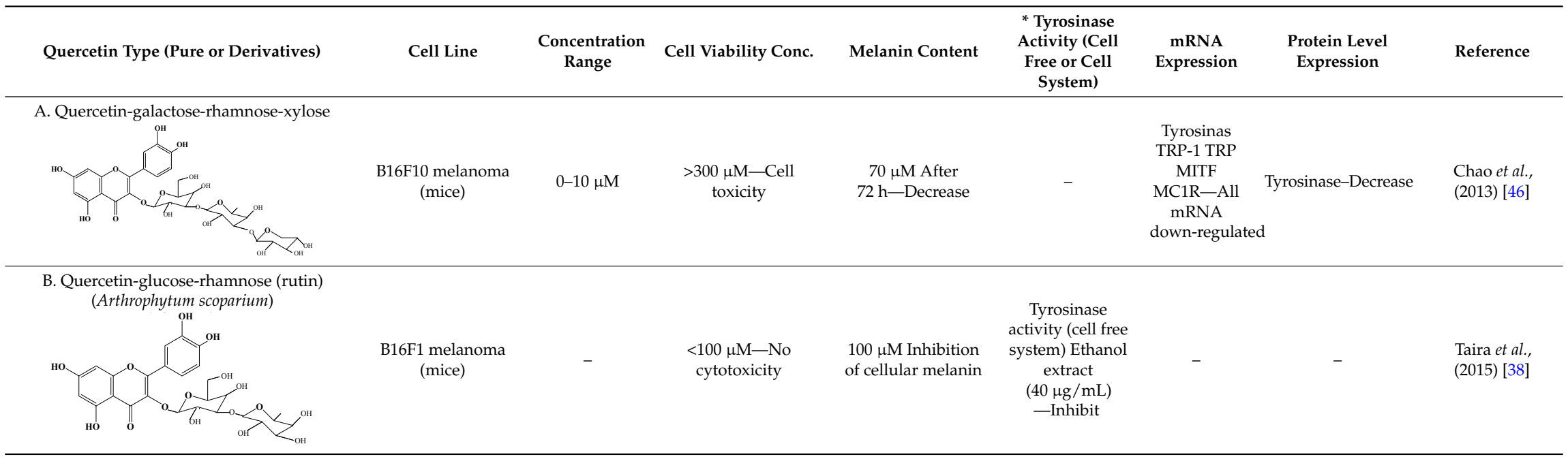

* Tyrosinase activity in intracellular (cell) system was significantly inhibited compared to that in a cell-free system. 


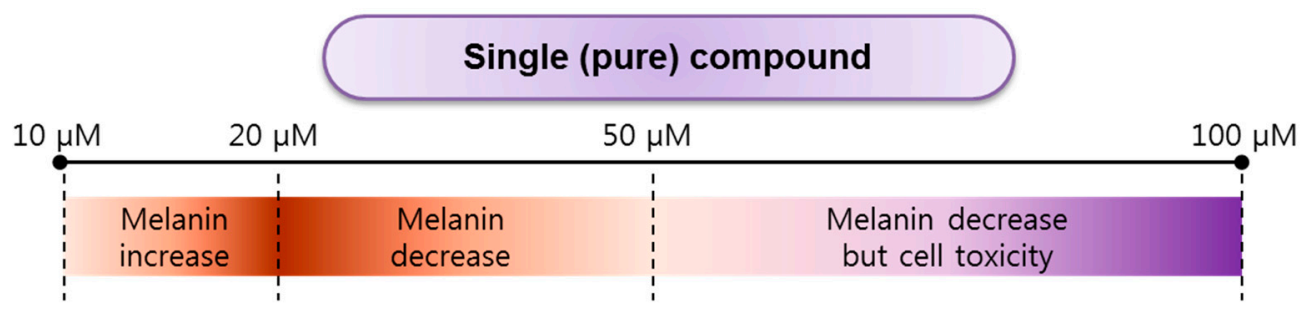

\section{Compounds in natural extracts}

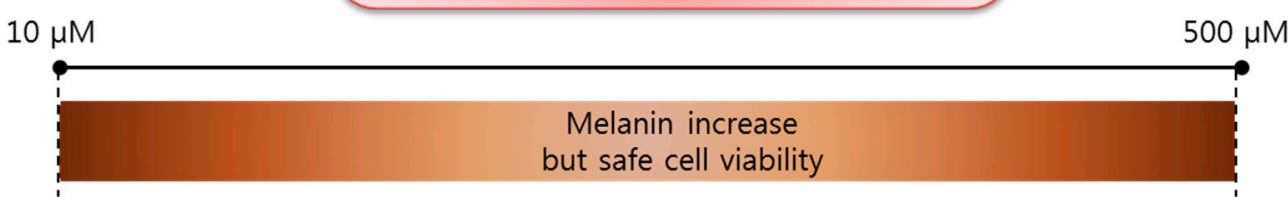

Figure 3. Concentration dependency of pure quercetin and natural quercetin in cell viability and anti-melanogenesis.

In summary, melanin content is not closely associated to tyrosinase activity or mRNA and protein expression levels [45]. However, Chao et al. (2013) reported that the melanin content is related to a decrease in tyrosinase activity, a downregulation of mRNA expression, and a decrease in protein expression levels [46]. Clearly, the anti-melanogenesis effect of quercetin is not clear and it remains to be determined whether it induces an increase or decrease in melanin content. It is possible that the effect depends on the quercetin concentration, and this should be tested in vitro using melanoma cells.

In order to evaluate the anti-melanogenesis effect of quercetin, in vivo analyses were performed using the zebrafish, useful for studies of melanogenic inhibitors or stimulators in terms of deformed morphologies or cell killing [47], because of its cost-effective and rapid analyses, its close physiological relevance to humans [48], and the establishment of a transgenic zebrafish for superficial skin ablation [49]. Chen et al. (2011) found that zebrafish larvae exhibit low toxicity owing to the radical oxygen scavenging properties of the antioxidant quercetin [42]. Mitochondrial ATPase as a target in zebrafish embryos modulates pigmentation in both melanocytes and melanoma cells [50]. Xenopus laevis pigment cell development can be tested using NSC 86153 compound but, unfortunately, not using quercetin [51]. However, an anti-melanogenesis effect of quercetin has not been observed in the zebrafish model. Therefore, our group recently tested zebrafish for an anti-melanogenic effect based on quercetin concentration, and found a negligible effect on hypo-pigmentation and cell mortality at quercetin concentrations $>100 \mu \mathrm{M}$. These data will be shown elsewhere.

\section{Concluding Remarks and Perspectives}

Based on a number of in vitro studies and relatively few in vivo studies, quercetin and its derivatives are not effective anti-melanogenesis agents. Pure quercetin, at $>50 \mu \mathrm{M}$, results in a decreased melanin content, while at 10-20 $\mu \mathrm{M}$, the melanin content increases in a concentration-dependent manner (Figure 3). Therefore, quercetin is not effective in cosmetic applications as a whitening ingredient. However, quercetin glycosides may be suitable for anti-melanogenesis purposes with no cytotoxicity. Other quercetin derivatives, including cyanidins, merit further tests of their anti-melanogenesis effects in zebrafish, humans, and other animals. To minimize cell toxicity, the use of vitamin $C$ and arbutin with quercetin has been suggested. The growing demand for whitening cosmetics has had a positive impact on the search for anti-melanogenesis ingredients. The consistent development of new and innovative agents drives market growth; hence, new assay techniques and delivery systems are being developed to improve the application of the agents in cosmetic products. 
Acknowledgments: This work was supported by the Human Resource Training Program for Regional Innovation and Creativity through the Ministry of Education and National Research Foundation of Korea (2015H1C1A1035883).

Author Contributions: Moon-Hee Choi and Hyun-Jae Shin equally contributed to the writing of the manuscript.

Conflicts of Interest: The authors declare no conflict of interest.

\section{Abbreviations}

\begin{tabular}{|c|c|}
\hline $\mathrm{ACTH}$ & adrenocorticotropin melanocyte stimulating hormone \\
\hline cAMP & cyclic adenosinemonophosphate \\
\hline cGMP & cyclic guanosine monophosphate \\
\hline CREB & cAMP-response element binding protein \\
\hline DCT & DOPA chrome tautomerase \\
\hline DHI & 5,6-dihydroxyindole \\
\hline DHICA & 5,6-dihydroxyindole-2-carboxylic acid \\
\hline DOPA & 3,4-dihydroxyphenylalanine \\
\hline ER & endoplasmic reticulum \\
\hline ERK & extracellular signal-regulated kinase \\
\hline HBTA & 5-hydroxyl-1,4-benzothiazinylalanine \\
\hline HMVII & human melanoma of vagina \\
\hline ICAQ & indole-2-carboxylic acid-5,6-quinone \\
\hline IQ & indole-5,6-quinone \\
\hline L-DOPA & L-3,4-dihydroxyphenylalanine \\
\hline LEF & lymphoid-enhancing factor \\
\hline MEK & methyl ethyl ketone \\
\hline MAP kinase & mitogen-activated protein kinase \\
\hline MART1 & melan-A, MC1R; melanocortin 1 receptor \\
\hline MC1R & melanocortin 1 receptor \\
\hline MITF & microphthalmia-associated transcription factor \\
\hline MTT & 3-(4,5-dimethylthiazol-2-yl)-2,5-diphenyltetrazolium bromide \\
\hline NHEM & normal human epidermal melanocytes \\
\hline $\mathrm{NO}$ & nitric oxide \\
\hline OA1 & ocular albinism type 1 \\
\hline PAR-2 & protease activated receptor 2 \\
\hline PKA & protein kinase A \\
\hline PKC- $\beta$ & protein kinase $C-\beta$ \\
\hline PMEL17 & Premelanosome protein 17 \\
\hline P13K & phosphoinositide 3-kinase \\
\hline SCF & stem cell factor \\
\hline TCA & tricarboxylic acid \\
\hline TRP-1 & tyrosinase-related protein 1 \\
\hline TRP-2 (DCT) & tyrosinase-related protein 2 (DOPA chrome tautomerase) \\
\hline TYR & tyrosinase \\
\hline UV & ultraviolet \\
\hline Wnt & wingless type \\
\hline
\end{tabular}

\section{References}

1. Taieb, A.; Cario-Andre, M.; Briganti, S.; Picardo, M. Inhibitors and enhancers of melanogenesis. In Melanins and Melanosomes; Borovansky, J., Riley, P.A., Eds.; Wiley-Blackwell: Weinheim, Germany, 2011; pp. 117-166.

2. Chang, T.S. An updated review of tyrosinase inhibitors. Int. J. Mol. Sci. 2009, 10, 2440-2475. [PubMed] 
3. Saxena, S.; Andersen, R.; Maibach, H.I. What do we know about depigmenting agents? Cosmet. Toilet. 2015, 130, 26-29.

4. Lin, J.Y.; Fisher, D.E. Melanocyte biology and skin pigmentation. Nature 2007, 445, 843-850. [CrossRef] [PubMed]

5. Chang, T.S. Natural melanogenesis inhibitors acting through the down-regulation of tyrosinase activity. Materials 2012, 5, 1661-1685. [CrossRef]

6. Solano, F.; Briganti, S.; Picardo, M.; Ghanem, G. Hypopigmenting agents: An updated review on biological, chemical and clinical aspects. Pigment Cell Res. 2006, 19, 550-571. [CrossRef] [PubMed]

7. Gillbro, J.M.; Olsson, M.J. The melanogenesis and mechanisms of skin-lightening agents-Existing and new approaches. Int. J. Cosmet. Sci. 2011, 33, 210-221. [CrossRef] [PubMed]

8. Simon, J.D.; Peles, D.; Wakamatsu, K.; Ito, S. Current challenges in understanding melanogenesis: Bridging chemistry, biological control, morphology, and function. Pigment Cell Melanoma Res. 2009, 22, 563-579. [CrossRef] [PubMed]

9. D’Lschia, M.; Wakamatsu, K.; Napolitano, A.; Briganti, S.; Garcia-Borron, J.C.; Kovacs, D.; Meredith, P.; Pezzella, A.; Picardo, M.; Sarna, T.; et al. Melanins and melanogenesis: Methods, standards, protocols. Pigment Cell Melanoma Res. 2013, 26, 616-633.

10. Haq, R.; Fisher, D.E. Targeting melanoma by small molecules: Challenges ahead. Pigment Cell Melanoma Res. 2013, 26, 464-469. [CrossRef] [PubMed]

11. Ma, X.P.; Sun, X.X. Melanin: Biosynthesis, Functions, and Health Effects; Nova Science Publishers, Inc.: New York, NY, USA, 2012; pp. 3-12.

12. Cao, H.H.; Tse, A.J.W.; Kwan, H.Y.; Yu, H.; Cheng, C.Y.; Su, T.; Fong, W.F.; Yu, Z.L. Quercetin exerts anti-melanoma activities and inhibits STAT3 signaling. Biochem. Pharmacol. 2014, 87, 424-434. [CrossRef] [PubMed]

13. Huang, H.C.; Chou, Y.C.; Wu, C.Y.; Chang, T.M. [8]-Ginerol inhibits melanogenesis in murine melanoma cells through down-regulation of the MAPK and PKA signal pathways. Biochem. Biophys. Res. Commun. 2013, 438, 375-381. [CrossRef] [PubMed]

14. Kim, K.N.; Yang, H.M.; Kang, S.M.; Kim, D.; Ahn, G.; Jeon, Y.J. Octaphlorethol A isolated from Ishige foliacea inhibits $\alpha$-MSH-stimulated induced melanogenesis via ERK pathway in B16F10 melanoma cells. Food Chem. Toxicol. 2013, 59, 521-526. [CrossRef] [PubMed]

15. Su, T.R.; Lin, J.J.; Tsai, C.C.; Huang, T.K.; Yang, Z.Y.; Wu, M.O.; Zheng, Y.Q.; Su, C.C.; Wu, Y.J. Inhibition of melanogenesis by gallic acid: Possible involvement of the PI3K/Akt, MEK/ERK and Wnt/ $\beta$-catenin signaling pathways in B16F10 cells. Int. J. Mol. Sci. 2013, 14, 20443-20458. [CrossRef] [PubMed]

16. Chung, K.W.; Jeong, H.O.; Jang, E.J.; Choi, Y.J.; Kim, D.H.; Kim, S.R.; Lee, K.J.; Lee, H.J.; Chun, P.; Byun, Y.; et al. Characterization of a small molecule inhibitor of melanogensis that inhibits tyrosinase activity and scavenges nitric oxide (NO). Biochim. Biophys. Acta-Gen. Subj. 2013, 1830, 4752-4761. [CrossRef] [PubMed]

17. Kim, S.S.; Kim, M.J.; Choi, Y.H.; Kim, B.K.; Kim, K.S.; Park, K.J.; Park, S.M.; Lee, N.H.; Hyun, C.G. Down-regulation of tyrosinase, TRP-1, TRP-2 and MITF expressions by citrus press-cakes in murine B16F10 melanoma. Asian Pac. J. Trop. Biomed. 2013, 3, 617-622. [CrossRef]

18. Kim, A.; Yang, Y.; Lee, M.S.; Yoo, Y.D.; Lee, H.G.; Lim, J.S. NDRG2 gene expression in B16F10 melanoma cells restrains melanogenesis via inhibition of Mitf expression. Pigment Cell Melanoma Res. 2008, 21, 653-664. [CrossRef] [PubMed]

19. Singh, S.K.; Sarkar, C.; Mallick, S.; Saha, B.; Bera, R.; Bhadra, R. Human placental lipid induces melanogenesis through p38 MAPK in B16F10 mouse melanoma. Pigment Cell Res. 2005, 18, 113-121. [CrossRef] [PubMed]

20. Ando, H.; Kondoh, H.; Ichihashi, M.; Hearing, V.J. Approaches to identify inhibitors of melanin biosynthesis via the quality control of tyrosinase (review). J. Investig. Dermatol. 2007, 127, 751-761. [CrossRef] [PubMed]

21. Takekoshi, S.; Nagata, H.; Kitatani, K. Flavonoids enhance melanogenesis in human melanoma cells. Tokai J. Exp. Clin. Med. 2014, 39, 116-121. [PubMed]

22. Xie, L.P.; Chen, Q.X.; Huang, H.; Wang, H.Z.; Zhang, R.Q. Inhibitory effects of some flavonoids on the activity of mushroom tyrosinase. Biochemistry 2003, 68, 598-602.

23. Boots, A.W.; Li, H.; Schins, R.P.F.; Duffin, R.; Heemskerk, J.W.M.; Bast, A.; Haenen, G.R.M.M. The quercetin paradox. Toxicol. Appl. Pharmacol. 2007, 222, 89-96. [CrossRef] [PubMed]

24. Heo, S.J.; Ko, S.C.; Cha, S.H.; Kang, D.H.; Park, H.S.; Choi, Y.U.; Kim, D.; Jung, W.K.; Jeon, Y.J. Effect of phlorotannins isolated from Ecklonia cava on melanogenesis and their protective effect against photo-oxidative stress induced by UV-B radiation. Toxicol. In Vitro 2009, 23, 1123-1130. [CrossRef] [PubMed] 
25. Siwarungson, N.; Ali, I.; Damsud, T. Comparative analysis of antioxidant and antimelanogenesis properties of three local guava (Psidium guajava L.) varieties of Thailand, via different extraction solvents. J. Food Meas. Charact. 2013, 7, 207-214. [CrossRef]

26. Takekoshi, S.; Matsuzaki, K.; Kitatani, K. Quercetin stimulates melanogenesis in hair follicle melanocyte of the mouse. Tokai J. Exp. Clin. Med. 2013, 38, 129-134. [PubMed]

27. Kim, Y.J. Hyperin and quercetin modulate oxidative stress-induced melanogenesis. Biol. Pharm. Bull. 2012, 35, 2023-2027. [CrossRef] [PubMed]

28. Chun, H.J.; Choi, W.H.; Baek, S.H.; Woo, W.H. Effect of quercetin on melanogenesis in melan-a melanocyte cells. Korean J. Pharmacogn. 2002, 33, 245-251.

29. Chun, H.J.; Hwang, S.G.; Kim, C.K.; Jeon, B.H.; Baek, S.H.; Woo, W.H. In vitro modulation of proliferation and melanization of B16/F10 melanoma cells by quercetin. Yakhak Hoeji 2002, 46, 75-80.

30. Cho, H.W.; Jung, W.S.; An, B.G.; Cho, J.H.; Jung, S.Y. Isolation of compounds having inhibitory activity toward tyrosinase from Receptaculum nelumbinis. Korean J. Pharmacogn. 2013, 44, 1-5.

31. Nagata, H.; Takekoshi, S.; Takeyama, R.; Homma, T.; Osamura, R.Y. Quercetin enhances melanogenesis by increasing the activity and synthesis of tyrosinase in human melanoma cells and normal human melanocytes. Pigment Cell Res. 2004, 17, 66-73. [CrossRef] [PubMed]

32. Takeyama, R.; Takekoshi, S.; Nagata, H.; Osamura, R.Y.; Kawana, S. Quercetin-induced melanogenesis in a reconstituted three-dimensional human epidermal model. J. Mol. Histol. 2004, 35, 157-165. [CrossRef] [PubMed]

33. Matsuyama, K.; Villareal, M.O.; Omri, A.E.; Han, J.; Kchouk, M.E.; Isoda, H. Effect of Tunisian Capparis spinosa L. extract on melanogenesis in B16 murine melanoma cells. J. Nat. Med. 2009, 63, 468-472. [CrossRef] [PubMed]

34. Yamauchi, K.; Mitsunaga, T.; Inagaki, M.; Suzuki, T. Synthesized quercetin derivatives stimulate melanogenesis in B16 melanoma cells by influencing the expression of melanin biosynthesis proteins MITF and p38 MAPK. Bioorg. Med. Chem. 2014, 22, 3331-3340. [CrossRef] [PubMed]

35. Yamauchi, K.; Mitsunaga, T.; Batubara, I. Novel quercetin glucosides from Helminthostachys zeylanica root and acceleratory activity of melanin biosynthesis. J. Nat. Med. 2013, 67, 369-374. [CrossRef] [PubMed]

36. Yamauchi, K.; Mitysunaga, T.; Batubara, I. Synthesis of quercetin glycosides and their melanogenesis stimulatory activity in B16 melanoma cells. Bioorg. Med. Chem. 2014, 22, 937-944. [CrossRef] [PubMed]

37. Fujii, T.; Saito, M. Inhibitory effect of quercetin isolated form Rose hip (Rosa canina L.) against melanogenesis by mouse melanoma cells. Biosci. Biotechnol. Biochem. 2009, 73, 1989-1993. [CrossRef] [PubMed]

38. Taira, J.; Tsuchida, E.; Uehara, M.; Ohhama, N.; Ohmine, W.; Ogi, T. The leaf extract of Mallotus japonicas and its major active constituent, rutin, suppressed on melanin production in murine B16F1 melanoma. Asian Pac. J. Trop. Biomed. 2015, 5, 819-823. [CrossRef]

39. An, S.M.; Kim, H.J.; Kim, J.E.; Boo, Y.C. Flavonoids, taxifolin and luteolin attenuate cellular melanogenesis despite increasing tyrosinase protein levels. Phytother. Res. 2008, 22, 1200-1207. [CrossRef] [PubMed]

40. Gómez-Cordovés, C.; Bartolomé, B.; Vieira, W.; Virador, V.M. Effects of wine phenolics and sorghum tannins on tyrosinase activity and growth of melanoma cells. J. Agric. Food Chem. 2001, 49, 1620-1624. [CrossRef] [PubMed]

41. Yang, Y.M.; Son, Y.O.; Lee, S.A.; Jeon, Y.M.; Lee, J.C. Quercetin inhibits $\alpha$-MSH-stimulated melanogenesis in B16F10 melanoma cells. Phytother. Res. 2011, 25, 1166-1173. [CrossRef] [PubMed]

42. Chen, Y.H.; Yang, Z.S.; Wen, C.C.; Chang, Y.S.; Wang, B.C.; Hsiao, C.A.; Shih, T.L. Evaluation of the structure-activity relationship of flavonoids as antioxidants and toxicants of zebrafish larvae. Food Chem. 2012, 134, 717-724. [CrossRef] [PubMed]

43. Arung, E.T.; Furuta, S.; Ishikawa, H.; Kusuma, I.W.; Shimizu, K.; Kondo, R. Anti-melanogenesis properties of quercetin- and its derivative-rich extract from Allium cepa. Food Chem. 2011, 124, 1024-1028. [CrossRef]

44. Masuda, M.; Itoh, K.; Murata, K.; Naruto, S.; Uwaya, A.; Isami, F.; Matsuda, H. Inhibitory effects of Morinda citrifolia extract and its constituents on melanogenesis in murine B16 melanoma cells. Biol. Pharm. Bull. 2012, 35, 78-83. [CrossRef] [PubMed]

45. Nakashima, S.; Matsuda, H.; Oda, Y.; Nakamura, S.; Xu, F.; Yoshikawa, M. Melanogenesis inhibitors from the desert plant Anastatica hierochuntica in B16 melanoma cells. Bioorg. Med. Chem. 2010, 18, 2337-2345. [CrossRef] [PubMed] 
46. Chao, H.C.; Najjaa, H.; Villareal, M.O.; Ksouri, R.; Han, J.; Neffati, M.; Isoda, H. Arthrophytum scoparium inhibits melanogenesis through the down-regulation of tyrosinase and melanogenic gene expressions in B16 melanoma cells. Exp. Dermatol. 2013, 22, 131-136. [CrossRef] [PubMed]

47. Choi, T.Y.; Kim, J.H.; Ko, D.H.; Kim, C.H.; Hwang, J.S.; Ahn, S.; Kim, S.Y.; Kim, C.D.; Lee, J.H.; Yoon, T.J. Zebrafish as a new model for phenotype-based screening of melanogenic regulatory compounds. Pigment Cell Res. 2007, 20, 120-127. [CrossRef] [PubMed]

48. Shiraki, T.; Kojima, D.; Fukada, Y. Light-induced body color change in developing zebrafish. Photochem. Photobiol. Sci. 2010, 9, 1498-1504. [CrossRef] [PubMed]

49. Chen, C.F.; Chu, C.Y.; Chen, T.H.; Lee, S.J.; Shen, C.N.; Hsiao, C.D. Establishment of a transgenic zebrafish line for superficial skin ablation and functional validation of apoptosis modulators in vivo. PLoS ONE 2011, 6, e20654. [CrossRef] [PubMed]

50. Jung, D.W.; Williams, D.; Khersonsky, S.M.; Kang, T.W.; Heidary, N.; Chang, Y.T.; Orlow, S.J. Identification of the F1F0 mitochondrial ATPase as a target for modulating skin pigmentation by screening a tagged triazine library in zebrafish. Mol. Biosyst. 2005, 1, 85-92. [PubMed]

51. Tomlinson, M.L.; Rejzek, M.; Fidock, M.; Field, R.A.; Wheeler, G.N. Chemical genomics identifies compounds affecting Xenopus laevis pigment cell development. Mol. Biosyst. 2009, 5, 376-384. [CrossRef] [PubMed]

(c) 2016 by the authors; licensee MDPI, Basel, Switzerland. This article is an open access article distributed under the terms and conditions of the Creative Commons Attribution (CC-BY) license (http://creativecommons.org/licenses/by/4.0/). 